\title{
Mediziner und Zellenlehre
}

\author{
Von Erwin H.AgKerknecht, Zürich
}

Mit Schwann hatten sich die mannigfachen Beobachtungen und Spekulationen über Zellen zu einer wissenschaftlichen Theorie der Grundelemente alles Lebenden verdichtet, wobei diese Grundelemente auch optisch nun ungefähr dem entsprachen, was wir heute unter dem Mikroskop sehen. In der großen Begeisterung, welche Schwanns Publikation auslöste, wurde es zuerst übersehen, daß seine Darstellung - großenteils dank seinem Bestreben, die Zellentstehung als Kristallisation zu sehen - in einem grundlegenden Punkt irrig war, dem der Zellbildung. Bekanntlich vertrat er die Vorstellung einer Zellbildung vorzugsweise aus einem amorphen «Cytoblastem » auf dem Weg über kleinste Kügelchen. Diese Frage wurde schließlich ebenfalls vorwiegend von Schülern von Joh. Müller, nämlich von Remak und Virchow aufgeklärt. Wir haben diese im Titel als «Mediziner» bezeichnet, weil sie der praktischen Medizin wesentlich näher standen, als ein Müller oder Schwann ${ }^{1}$. Es ist kein Zufall, daß gerade dieses Problem von «Medizinern» angepackt wurde. Nur nach seiner Lösung konnte nämlich die Medizin wirklich von der Zellenlehre profitieren.

Es hatte sich sofort im Anschluß an Schwanns Entdeckung und lange vor Virchow eine Zellularpathologie entwickelt (siehe die Werke von Joh. Müller, Henle, Gluge, Güterbock, Vogel, Donné, Lebert usw.) ${ }^{1 a}$. Die Mediziner waren glücklich, endlich wieder ein Grundelement zu haben, auf das sie alle Pathologie reduzieren konnten. Seit Verschwinden der Säftelehre hatte ihnen ein solches gefehlt. Ihre zellularpathologischen Bemühungen scheiterten aber gerade an der Schwannschen Zellbildungslehre, die sie die Dinge direkt auf dem Kopf sehen ließ, d.h. zerfallende Zellen erschienen ihnen als junge, junge Zellen als alte. Wir erinnern z.B. an Gluges «Entzündungskugeln» und «Sternzellen». Rokitansky baute gar eine falsche Humoralpathologie auf Schwanns Irrtum auf!

Wie immer in der Geschichte der Zellenlehre wurde auch das Zellteilungsproblem zuerst korrekt von den Botanikern angefaßt, die allerdings auch

${ }^{1}$ Für mehr über die praktische Haltung der Müller-Schönlein-Schüler Virchow, Remak und Lebert siehe E.H. Ackerknecht, Zellulartheorie und Therapie, Praxis 1968.

${ }^{1 a}$ E. H. ACKERKNECHT, Rudolf Virchow, Stuttgart 1957, S. 61. 
mit klareren Verhältnissen arbeiteten. Nach den Arbeiten von Mohl (1835), Unger (1841) und Nägeli (1844) stand es ziemlich fest, daß Pflanzenzellen nur aus Pflanzenzellen entspringen ${ }^{2}$.

Das Verdienst diese Tatsache auch für die tierische Zelle festgehalten und damit auf die ganze belebte Natur ausgedehnt zu haben, gebührt John Goodsir (1845), Robert Remak (1852) und Rudolf Virchow (1855). Dieser Einsicht zur Anerkennung zu verhelfen war durchaus nicht einfach, da sich die Schwannsche Lehre einer außerordentlichen Beliebtheit nicht nur in der gelehrten Welt im allgemeinen erfreute, sondern auch bei denen, die berufen waren, sie zu reformieren. Weder G. Valentin noch J.Vogel äußern z.B. die geringsten Zweifel an der Schwannschen Zellbildungstheorie in ihren grundlegenden Beiträgen zur Gewebelehre in Rud. Wagners Handwörterbuch der Physiologie (Band I, Braunschweig 1842). In der vierten Auflage seines Handbuches (1844) wiederholt Joh. Müller kritiklos die Lehre seines Schülers Schwann. Reichert, der (Entwicklungsleben, Berlin 1840) den tatsächlichen Verhältnissen recht nahe gekommen war, kommt später wieder (z.B. Müller, Arch.1851, S.525) davon ab. Es ist ungemein bezeichnend, daß 1855 Kölliker, der seit 1844 an der Schwannschen Cytoblastemlehre gezweifelt hatte, sich immer noch außer Stande erklärte Remak und Virchow in ihrer radikalen Ablehnung derselben zu folgen ${ }^{3}$. Während sich K. Ludwig 1856 um eine Entscheidung drückte, indem er alle Zellbildungslehren bezweifelte ${ }^{4}$, hingen so ausgezeichnete Histologen wie Henle und J. Gerlach 1860 immer noch dem Blastem an ${ }^{5}$.

Von unsern drei Pionieren ist JoHN GoodsIR (1814-1867) seit 1842 Anatomieprofessor in Edinburgh und durchaus kein unbedeutender Mann, doch relativ der Unbedeutendste. Er hat sehr wenig publiziert, hat aber zweifellos Virchow, der ihn 1852 zitiert, beeinflußt, nicht nur bezüglich des Zellursprungs, sondern auch bezüglich der Zelle als Lebens- und Krankheitseinheit im Gegensatz zum Gefäß. In seinem Buch von 1845 (Anatomical and Pathological Observations) sagt er ausdrücklich, daß die Zelle aus dem Kern entstamme. Er erwähnt allerdings auch noch einmal beiläufig das

${ }^{2}$ H. von MoHL, Über die Vermehrung von Pflanzenzellen durch Teilung, Tübingen 1835. C. NÄGELI, Zellkern, Zellbildung und Zellteilung, Z. wiss. Botanik, Heft 1, 1844, 3 und 4 1846. - F. Unger, Linaea 1841, S. 389, siehe auch O. Hertwig, Die Zelle, Jena 1893, Kap. I und II.

3 Handbuch der Gewebelehre des Menschen, 2. Auflage, Leipzig, 1855, S. 17.

4 Lehrbuch der Physiologie des Menschen, Band II, S.161.

5 Handbuch der Gewebelehre des menschlichen Körpers, Wien 1860, S.17. 
Blastem ${ }^{6}$. Auf jeden Fall fand Goodsir in den vierziger Jahren kein Echo, teils wohl weil er die neue Einsicht zu kurz, zu abstrakt und ohne Details vorbrachte, teils auch weil die Zeit noch nicht reif war.

Bei Robert ReMaK (1815-1865) können wir dagegen sehr schön verfolgen, wie die Überzeugung von der Nicht-Existenz des Blastems langsam heranreifte und an dem verschiedenartigsten Material bewiesen wurde. Wir wissen glücklicherweise seit der außerordentlich verdienstvollen Arbeit von Bruno Kisch etwas mehr über diese tragische und merkwürdige Figur, diesen deutschen Juden, der seinen Glauben nicht aufgeben, dabei auch noch ausgerechnet als Pole gelten wollte und der darum trotz wahrhaft genialer Begabung sein ganzes kurzes Leben lang zurückgesetzt und nicht anerkannt wurde $^{7}$. Dabei hat Remak zwischen 1836 und 1843 einige der wichtigsten Tatsachen der Neuro-Histologie entdeckt (Achsencylinder, marklose sympathische Faser, ganglionärer Ursprung der Nervenfaser). Er hat zwischen 1843 und 1854 in der Embryologie dem von K.E.v.Baer und C.F. Wolff Geleisteten durchaus Ebenbürtiges an die Seite gestellt und dabei eben auch den Zellursprung aufgeklärt. Er hat in den letzten 10 Jahren seines Lebens die moderne Galvanotherapie begründet.

1841 beobachtete Remak zum erstenmal die Zellteilung im embryonalen Blutkörperchen. Kurz danach sah er sie auch an embryonalen Hirnzellen und im Krebs ${ }^{8}$. Wie aus seinem im folgenden Jahr erschienenen Referat in Cannstatt's Jahresbericht zu ersehen ist, stimmten ihn diese Beobachtungen bereits damals der Cytoblastemtheorie gegenüber mißtrauisch, die er auch wegen ihrer Analogie mit der unmöglichen Urzeugung wenig schätzte. Höchst vernünftigerweise fühlte er sich aber noch nicht berechtigt, dieselbe völlig zu verwerfen ${ }^{9}$. Im selben Jahr sah übrigens auch K. Bergmann, daß die Furchung Zellteilung war, kam aber nicht mehr auf das Problem zurück ${ }^{10}$. Im Jahr 1845 veröffentlichte Remak wiederum Teilungsbeobachtungen an der embryonalen Muskelzelle, welche gegen das Cytoblastem sprachen, zögerte aber immer noch endgültig Stellung zu nehmen ${ }^{11}$. Zwischen 1850 und 1852 häuften sich seine Beobachtungen aber derart, daß

6 S. 25 und 32.

7 Forgotten Leaders of Medicine, Philadelphia 1954, S. 227-296.

8 Med.Ver.Ztg. 1941, Nr. 27. - Arch. Anat. Physiol. wiss. Med.1841, S. 513. - Cannstatt's Jahrbuch über die Fortschritte der Medizin für 1841, Erlangen 1842, S. 9.

9 Cannstatt's Jahresbuch, S. 8-9.

10 Arch.Anat. Physiol.wiss. Med.1841, S. 89.

11 Frorieps Not. 35 (1845) 305. 
er dann 1852 mit den folgenden völlig eindeutigen und kraftvollen Ausführungen hervortrat ${ }^{12}$.

«Mir selbst war die extrazellulare Entstehung tierischer Zellen, seit dem Bekanntwerden der Zellentheorie, ebenso unwahrscheinlich, wie die 'Generatio aequivoca' der Organismen. Aus diesen Zweifeln entsprangen meine Beobachtungen über die Vermehrung der Blutzellen durch Teilung bei Embryonen von Vögeln und Säugetieren und über die Längsteilung der durch Verlängerung von Zellen entstehenden quergestreiften Muskelfasern (Muskelprimitivbündel) bei Froschlarven (Fror. N. Notizen 1845 Septbr. Nr. 768). Seitdem habe ich diese Beobachtungen an Froschlarven fortgesetzt, bei welchen es möglich ist, die Entstehungsgeschichte der Gewebe bis auf die Furchung zurückzuführen. Doch ist es mir erst im Frühling dieses Jahres (1851) gelungen, zu ermitteln, daß sämtliche aus der Furchung hervorgehende Embryonalzellen sich bei ihrem Übergange in die Gewebe durch Teilung vermehren.

Diese Ergebnisse haben zur Pathologie eine ebenso nahe Beziehung wie zur Physiologie. Es kann kaum noch bestritten werden, daß die pathologischen Gewebeformen nur Varianten der normalen embryonischen Entwickelungstypen bilden und es ist nicht wahrscheinlich, daß sie das Vorrecht der extrazellularen Entstehung von Zellen besitzen sollten. Die sogenannte 'Organisation der plastischen Exsudate' und die früheste Bildungsgeschichte der krankhaften Geschwülste bedarf in dieser Hinsicht einer Prüfung. Gestützt auf die Bestätigung, welche meine vieljährigen Zweifel erfahren, wage ich die Vermutung auszusprechen, daß die pathologischen Gewebe ebensowenig wie die normalen in einem extrazellularen Zytoblastem sich bilden, sondern Abkömmlinge oder Erzeugnisse normaler Gewebe des Organismus sind.»

1854 setzte er diese Erwägungen in einer Krebsarbeit ${ }^{13}$ fort und $1855^{14}$ berichtete er relativ loyal über die respektiven Prioritäten in dem Gesamtverlauf der Diskussion, wobei er allerdings Virchow, der inzwischen zahlreiches pathologisches Material zur Diskussion beigetragen hatte, nicht erwähnt. Dazu war er formal insofern berechtigt, als er nur über die Entwicklung im Rahmen der Embryologie referierte.

12 Über die extracelluläre Entstehung thierischer Zellen, Arch. Anat. Physiol. wiss. Med.1852, S. $49,57$.

13 Deutsche Klinik 6 (1854) 170-174.

14 Untersuchungen über die Entwicklung der Wirbeltiere, Berlin 1855, S.164-179. 
Warum werden wir nun im Folgenden überhaupt noch über RudoLF Virchow (1821-1902) sprechen, nachdem Remaks Priorität so zweifellos feststeht? Bestimmt nicht, weil so viele in den letzten 100 Jahren Virchow wegen seines Schlagworts «Omnis cellula e cellula» das ausschließliche Recht auf die Entdeckung gegeben und Remaks Verdienste verschwiegen haben! Es muß aber doch u.a. anerkannt werden, daß Virchow zu seinen Schlußfolgerungen mit einem völlig anderen Material gelangt ist. Und daß er den Grundsatz, daß eine Zelle von der andern abstammt wie kein anderer durchgefochten und schließlich durchgesetzt hat. Im Gegensatz zu Remak brauchen wir hier nicht auf Virchows Bedeutung im allgemeinen und seine zahlreichen selbständigen großen Leistungen im Lauf seines langen Lebens hinweisen, da wir dies an anderer Stelle ausführlich getan haben und wohl auch als bekannt voraussetzen dürfen.

In einer Rede, die der 24jährige Virchow bei der Stiftungsfeier der Pepinière hielt (1845), sehen wir ihn zwar als Gegner der Urzeugung, aber als Anhänger der Schwannschen Blastemlehre ${ }^{15}$. Virchow war 6 Jahre jünger als Remak, und die Zweifel an der Schleiden-Schwannschen Theorie kamen ihm auch $6 \mathrm{Jahre} \mathrm{später,} \mathrm{nämlich} \mathrm{1847.} \mathrm{Er} \mathrm{schreibt} \mathrm{im} \mathrm{1.} \mathrm{Band} \mathrm{seines}$ Archivs (S.484), daß «die Schleiden-Schwannsche Theorie in der alten Form nicht länger zu halten ist »; er hängt dieser Theorie dann aber mangels einer besseren doch noch mindestens sieben Jahre lang an! Z.B. beschreibt er im selben 1.Band des Archivs den Ursprung des Krebses aus dem Blastem (S.110, 218). Und er versteht noch gar nicht, daß er mit seiner gleichzeitigen ausgedehnten Polemik gegen die sogenannten «Entzündungskugeln» (S.141 ff.), die sich auf Arbeiten seines Freundes Reinhardt stützt und zeigt, daß diese nicht junge sondern zerfallende Zellen sind, bereits die pathologisch-anatomische Anwendung der Blastemtheorie demoliert. So vertritt er auch im 5. Band des Archivs (1853) immer noch das Blastem (S. 239), obwohl er bereits 1851 die Kernteilung im Krebs beschreibt (Archiv 3, S.197). Auch 1852 läßt er noch das Bindegewebe aus dem Blastem entstehen ${ }^{16}$. Im selben Jahr spricht er allerdings aus, daß neue Zellen das Resultat einer Zellteilung sind, ohne das Blastem zu erwähnen ${ }^{17}$.1854 sagt er im 1. Band seines Handbuchs der speziellen Pathologie und Therapie etwas verklausuliert dasselbe ${ }^{18}$, zögert aber ausdrücklich Remak in seiner

15 Gedruckt in Virchows Arch. 18819071.

16 Verh. physik.-med. Ges. 2 Würzburg (1852) 315.

17 Virchows Arch.4 (1852) 377.

18 «Es gibt nur Leben durch direkte Nachfolge», S. 3. 
völligen Ablehnung des Blastems zu folgen ${ }^{19}$. Erst 1855 ringt er sich in dem berühmten Leitartikel «Zellularpathologie» im 8. Band des Archivs zu der bekannten Formulierung «Omnis cellula a cellula» durch, und nennt nun auch gleich ebenso unbescheiden wie zutreffend seine auf dieser neuen Zelltheorie basierende neue Zellularpathologie «die Pathologie der Zukunft».

Es ist nicht uninteressant, daß Virchow sogar noch 1856 sich zwar gegen die freie Zellbildung, aber in einer eigentümlich verklausulierten Form aussprach $^{20}$. Zusammenfassend möchte ich wiederholen, was ich vor einigen Jahren über Virchows Leistung gesagt habe:

«Nachdem wir Virchows Vorgänger gebührend gewürdigt haben (was er übrigens gelegentlich selbst tat), muß anerkannt werden, daß er seine Schlußfolgerung unabhängig, induktiv und auf seine eigene Weise erreichte. Offenbar ist dies der Grund, warum er sie so spät erreichte. Aber wahrscheinlich ist es auch die Ursache dafür, daß seine Feststellungen einen unvorhergesehenen Einfluß auf die Menge der Ärzte hatte. Virchow besaß nicht das ausgedehnte Interesse an der vergleichenden Anatomie oder der Embryologie, das die anderen Schüler von Johannes Müller oder einen John Goodsir charakterisierte; wenn er es besessen hätte, würde er wahrscheinlich seine Entdeckung früher gemacht haben, doch hätte er dann eine ebenso geringe Reaktion in der Ärzteschaft hervorgerufen, wie es Remak tat. Da er fast ausschließlich mit pathologischem Material arbeitete, erreichte er seine Folgerungen später; doch sie waren von größerer Bedeutung und für den Mediziner unmittelbar anwendbar. Zu der Tatsache, daß Virchows Funde mehr Bedeutung besaßen, kam hinzu, daß er sie mit jenem unermüdlichen Eifer und der fast unheimlichen Energie verbreitete, in denen er niemals übertroffen wurde. ${ }^{21}$

1863 scheint die Sache dann entschieden gewesen zu sein, denn Kölliker trat nun in der 4.Auflage seiner Gewebelehre rückhaltlos für die neue Zelltheorie ein und fand dementsprechend in seinen historischen Überblicken einen bedeutend größeren Beitrag zum Aufbau derselben aus der Feder Köllikers, als in früheren Auflagen seines Buches. Die vorhergehende Beschreibung der Überwindung der Blastemtheorie zeigt wohl, daß auch große Männer - und dies gereicht ihnen keineswegs zur Schande - oft keineswegs schlagartig das Neue entdecken, sondern sich langsam und mühselig mit Fehlern und Schwankungen dazu durchringen. Daß sich ihnen

19 S. 329.

20 Ges. Abh.wiss. Med., Frankfurt 1856, S. 327.

21 E.H. ACKerKNeGht, 1.c., S.70. 
selbst rückblickend der Prozeß meist viel gradliniger darstellt, ist verständlich, aber kein Grund, ihnen auf diesem Wege zu folgen.

In die Jahre der Entscheidung über die Zellvermehrung fallen auch die Diskussionen über die Zellelemente. Da die Zellehre aus der Botanik stammt, war die Zellwand anfangs stark überschätzt worden (z.B. auch noch in Virchows Zellular-Pathologie von 1858). Bischoff wies bereits 1842 (Entwicklungsgeschichte des Kaninchens), Kölliker 1844 (Entwicklungsgeschichte der Cephalopoden) darauf hin, daß tierische Zellen auch ohne Membrane existieren könnten. Purkinje hatte 1840 den Ausdruck Protoplasma geprägt: Hugo von Mohl verwendete ihn 1846 in unserem Sinn für die Pflanzenzelle, Remak übertrug ihn 1852 auf die tierische Zelle. Damit war der Weg dann frei für Max Schultzes moderne Zelldefinition im Archiv für Anatomie und Physiologie von 1861 : «Die Zelle ist ein Klümpchen Protoplasma mit einem Kern.»

Die Arbeiten von Remak, Virchow u.a. hatten die Frage des Verlaufs der Zellteilung nur teilweise beantwortet. Wiederum waren es die Botaniker (Russow, Schneider, Bütschli, besonders Strasburger 1875) die zuerst demonstrierten wie Zellteilung vor allem Kernteilung ist. Flemming, der seit 1878 an dem Problem arbeitete, zog 1882 die Konsequenz auch für das Tierreich und ergänzte das «Omnis cellula e cellula» durch den Satz: «Omnis nucleus e nucleo. » ${ }^{22}$ Die weiteren Probleme der Kernteilung hängen aufs engste mit dem Befruchtungsprozeß zusammen und werden Gegenstand einer anderen Untersuchung bilden müssen.

'22 W. Flemming, Zellsubstanz, Kern und Zellteilung, Leipzig 1882. 